\title{
Slow gait speed is associated with reduction in health status in older ambulatory Chinese: A study for comprehensive geriatric assessment
}

\section{Ping Zeng}

The MOH Key Laboratory of Geriatrics, Beijing Hospital, National Center of Gerontology https://orcid.org/0000-0003-2774-2533

\section{Yalun Dai}

The MOH Key Laboratory of Geriatrics, Beijing Hospital, National Center of Gerontology

\section{Yiwen Han}

The MOH Key Laboratory of Geriatrics, Beijing Hospital, National Center of Gerontology

\section{Jing Pang}

The MOH Key Laboratory of Geriatrics, Beijing Hospital, National Center of Gerontology

\section{Huan Gong}

The MOH Key Laboratory of Geriatrics, Beijing Hospital, National Center of Gerontology

Jian Li

The MOH Key Laboratory of Geriatrics, Beijing Hospital, National Center of Gerontology

Tiemei Zhang ( $\nabla$ tmzhang126@126.com )

The MOH Key Laboratory of Geriatrics, Beijing Hospital, National Center of Gerontology

\section{Research article}

Keywords: Gait speed, Physical performance, the Geriatric Depression Scale, Mini-mental State Examination, Co-morbidity, Sarcopenia

Posted Date: July 3rd, 2019

DOI: https://doi.org/10.21203/rs.2.10879/v1

License: (c) (1) This work is licensed under a Creative Commons Attribution 4.0 International License. Read Full License 


\section{Abstract}

Background: Gait speed (GS) is well recognized as a comprehensive index of health status in older people. Nationwide survey data in China have been used to measure the association between slow GS and health status in order to emphasize the importance of measuring GS to detect a decline in health status in older ambulatory Chinese. Methods: A group of 2677 male and 2668 female older (aged $\geq 60$ ) adults, who could perform the GS test, were selected as study subjects.GS was measured by subjects walking across and back over a $10-\mathrm{m}$ course. Gait speed less than $20 \%$ that of a reference population $(<0.7 \mathrm{~m} / \mathrm{s})$ was used as the threshold of slow GS. Results: Slow GS was associated with higher comorbidity, polypharmacy, more medical expenses, increased needs for care and hospitalization, worse ranking on the Geriatric Depression Scale (GDS), and decreased physical and cognitive functions. In logistic regression model, higher education level $(\mathrm{OR}=0.93,0.88-0.98)$, alcohol drinking $(\mathrm{OR}=0.82,0.71$ 0.95), scores on Mini-mental State Examination ( $\mathrm{OR}=0.95,0.93-0.96)$, doing housework $(\mathrm{OR}=0.51,0.45$ $0.57)$, and having a regular daily routine $(\mathrm{OR}=0.71,0.51-0.98)$ were independently associated with faster GS; while co-morbidity ( $O R=1.79,1.59-2.03)$, expressed disinterest in life ( $O R=1.22,1.12-1.32)$, and dissatisfaction with personal health $(\mathrm{OR}=1.22,1.13-1.33)$ were associated with slow $G S$. The AUC value on ROC analysis was 0.72 for the selected variables. Conclusions: Slow GS is associated with poorer health status in Chinese older people. Measuring GS may be a quick and inexpensive tool to help identify individuals with declines in health status and who might benefit from targeted intervention.

\section{Introduction}

Background: Gait speed (GS) is well recognized as a comprehensive index of health status in older people. Nationwide survey data in China have been used to measure the association between slow GS and health status in order to emphasize the importance of measuring GS to detect a decline in health status in older ambulatory Chinese.

Methods: A group of 2677 male and 2668 female older (aged $\geq 60$ ) adults, who could perform the GS test, were selected as study subjects.GS was measured by subjects walking across and back over a 10-m course. Gait speed less than $20 \%$ that of a reference population $(<0.7 \mathrm{~m} / \mathrm{s})$ was used as the threshold of slow GS.

Results:Slow GS was associated with higher co-morbidity, polypharmacy, more medical expenses, increased needs for care and hospitalization, worse ranking on the Geriatric Depression Scale (GDS), and decreased physical and cognitive functions. In logistic regression model, higher education level $(O R=0.93$, 0.88-0.98), alcohol drinking ( $O R=0.82,0.71-0.95)$, scores on Mini-mental State Examination $(O R=0.95$, $0.93-0.96)$, doing housework $(O R=0.51,0.45-0.57)$, and having a regular daily routine $(O R=0.71,0.51-0.98)$ were independently associated with faster GS; while co-morbidity $(O R=1.79,1.59-2.03)$, expressed disinterest in life $(O R=1.22,1.12-1.32)$, and dissatisfaction with personal health $(O R=1.22,1.13-1.33)$ were associated with slow GS. The AUC value on $R O C$ analysis was 0.72 for the selected variables. 
Conclusions: Slow GS is associated with poorer health status in Chinese older people. Measuring GS may be a quick and inexpensive tool to help identify individuals with declines in health status and who might benefit from targeted intervention.

Keywords: Gait speed, Physcial performance, the Geriatric Depression Scale, Mini-mental State Examination, Co-morbidity, Sarcopenia

\section{Background}

China has a rapidly increasing number of older people, many of whom have disabilities or reduced cognitive function. This situation has highlighted the need for an effective strategy to deal with the burden of caring for an ageing population, which means maintaining physical and mental function to enable the older people to live independently until their end stage of life [1-3]. For these reasons, sarcopenia has become an active research topic in recent years due to the association between poor health status and the adverse outcomes of sarcopenia, such as low physical performance, increased frequency of disability, lesser quality of life and higher health care costs and mortality [4-7]. To reduce these adverse outcomes that are challenges of ageing, the population at risk should be monitored and receive targeted intervention measures when needed $[8,9]$.

As one of the main components for sarcopenia, walking requires considerable energy and coordination, and walking speed may reflect the multi-systemic well-being of older people $[3,10]$. The speed of walking is closely related to the strength of the body, balance, endurance, and cognitive and mental status. Therefore, gait speed (GS) can be considered as a comprehensive index that reflects the overall health status of an individual and serves as a marker of physiological reserve [10,11]. Some studies have suggested that GS can be used to identify people at high risk of adverse health outcomes [12,13]. In the reivised European consensus on definition and diagnosis of Sarcopenia, slow GS has been considered as indicative of servere sarcopenia [14]. The opposite of slow walking is fast walking. In another Chinese population, Woo et al. found that fast walking speeds (>1.39 m/s) maybe a sign of successful ageing, since the variables significantly associated with fast walkers are similar to the attributes of successful ageing [3].

Compared with sarcopenia, GS measurements are more feasible to obtain in older populations; they are quick, inexpensive, and easy to administer. Surprisingly, measuring GS is not widely used in evaluating the heath of older people in China, perhaps because of a lack of sufficient evidence to document its importance. To fill this gap, this study uses nationwide survey data to examine the association between slow GS and health status in a population of older Chinese ambulatory adults and to explore the use of GS as a measure of decline in health status and a signal for early intervention.

China has a rapidly increasing number of older people, many of whom have disabilities or reduced cognitive function. This situation has highlighted the need for an effective strategy to deal with the burden of caring for an ageing population, which means maintaining physical and mental function to enable the older people to live independently until their end stage of life [1-3]. For these reasons, 
sarcopenia has become an active research topic in recent years due to the association between poor health status and the adverse outcomes of sarcopenia, such as low physical performance, increased frequency of disability, lesser quality of life and higher health care costs and mortality [4-7]. To reduce these adverse outcomes that are challenges of ageing, the population at risk should be monitored and receive targeted intervention measures when needed $[8,9]$.

As one of the main components for sarcopenia, walking requires considerable energy and coordination, and walking speed may reflect the multi-systemic well-being of older people $[3,10]$. The speed of walking is closely related to the strength of the body, balance, endurance, and cognitive and mental status. Therefore, gait speed (GS) can be considered as a comprehensive index that reflects the overall health status of an individual and serves as a marker of physiological reserve [10,11]. Some studies have suggested that GS can be used to identify people at high risk of adverse health outcomes $[12,13]$. In the reivised European consensus on definition and diagnosis of Sarcopenia, slow GS has been considered as indicative of servere sarcopenia [14]. The opposite of slow walking is fast walking. In another Chinese population, Woo et al. found that fast walking speeds (>1.39 m/s) maybe a sign of successful ageing, since the variables significantly associated with fast walkers are similar to the attributes of successful ageing [3].

Compared with sarcopenia, GS measurements are more feasible to obtain in older populations; they are quick, inexpensive, and easy to administer. Surprisingly, measuring GS is not widely used in evaluating the heath of older people in China, perhaps because of a lack of sufficient evidence to document its importance. To fill this gap, this study uses nationwide survey data to examine the association between slow GS and health status in a population of older Chinese ambulatory adults and to explore the use of GS as a measure of decline in health status and a signal for early intervention.

\section{Methods}

\section{Participants}

The data were drawn from a nation-wide cross-sectional survey conducted in 2011 for evaluating the health status of older Chinese. The investigators and study participants came from 13 hospitals in six administrative regions of China. The study subjects, who were at least 60 years old, were community residents who were patients in regular health examination centers, outpatient departments, and wards of the participating hospitals. Only subjects who could perform the walking test were included in this analysis.

Questionnaires were used to collect information on demographics, lifestyle, and medical history, including receipt of care. Individuals with two or more common diseases, such as hypertension, diabetes, cardiovascular diseases, coronary heart disease, chronic obstructive pulmonary disease (COPD), osteoarthritis, and cancer were considered to have co-morbidity. Activities of daily living (ADL) and instrumental activities of daily life (IADL) were measured by standardized instruments. Cognitive function and psychological status were evaluated by Mini-mental State Examination (MMSE) and Geriatric 
Depression Scale (GDS), respectively. Questions about "whether interestedin life" and "self-rated satisfaction with health" were included in this study to add information about psychological status in a Chinese cultural context. "Doing housework" and "Having a regular daily routine" were also included because they may indicate the psychological status and life attitude of the individual. Doing housework was answered affirmatively when a person was doing work such as house cleaning, cooking, taking care grandchildren, and described the frequency as "often". Having a regular daily routine was defined as a person who has a regular daily time schedule. The other life style variables included currently smoking ( $\geq 5$ cigarettes/day); regular drinking of alcohol (at least once a week); and engaging in physical exercise (moderate or more, at least once a week and lasting for30 min or more). The interviewers were trained before the survey was administered, the subjects' informed consent was obtained to use their information in this study, and the study was approved by the Ethics Committee of Beijing Hospital, Ministry of Health.

\section{Measurement}

The GS was evaluated by measuring the time for the subject to walk 20 meters. Participants walked at their usual pace from a standing start and continued walking straight forward for 10 meters, at which point they made a U-turn and returned to the starting line. Slow GS was defined as a value in the $20^{\text {th }}$ percentile of GS measured in the old participants in this study [15], which was $<0.7 \mathrm{~m} / \mathrm{s}$.

\section{Statistical Analysis}

Descriptive statistics (i.e., means, SDs and proportions) were used to characterize the demographics and measured variables of the subjects. The differences of continuous variables were compared using analysis of variance with two factors. Frequency data were compared by Mantel-Haenszel statistics to remove the confounding influence. Logistic regression (forward selection method) was performed to estimate the adjusted odds ratios and the $95 \%$ confidence interval $(95 \% \mathrm{Cl})$ of the variable associated with slow GS. Using predicted probabilities, receiver-operating characteristic $(R O C)$ curves were constructed to evaluate the discriminative ability of the model. The area under the ROC curve (AUC) was used to test the concordance of predictive values with actual outcome. The statistical analyses were carried out using SAS software and $P<0.05$ was considered to be statistically significant.

\section{Results}

The characteristics of normal GS and slow GS groups are shown in Table 1 (in Supplementary Files). 2677 men and 2668 women were included in the study. The mean age in the slow GS group about three years older than the mean age of the normal GS group, but this difference was not statistically significant. The mean values of GS for each group was statistically significant $0.5 \mathrm{~m} / \mathrm{s}$ slower in the slow GS group than in the normal GS group. The people with slow GS had lower education level, had more diseases, took more kinds of medicines, and spent roughly twice as money on health care as people in the normal GS group. There were more people (about 10\%-20\%) in slow GS group needing care because of illness and who had been hospitalized during the past year. They had lower ADL and IADL 
performance, lower Mini-mental State Examination (MMSE) scores, more symptoms of depression, and fewer people ( $10 \%$ less) who could perform the 5 -time chair stand test.

After adjusting for age and gender, a significant increase in the prevalence of common diseases was found in the subjects with slow GS (Figure 1 in Supplementary Files). The differences ranged from $10 \%$ to $18 \%$ higher. Table 2 shows that higher education level $(O R=0.93,0.88-0.98)$, drinking alcohol regularly $(O R=0.82,0.71-0.95)$, better scores on MMSE $(O R=0.95,0.93-0.96)$, doing housework $(O R=0.51,0.45$ $0.57)$, and following a regular daily routine $(O R=0.71,0.51-0.98)$ were independently associated with faster GS. In contrast, co-morbidity $(O R=1.79,1.59-2.03)$, expressed disinterest in life $(O R=1.22,1.12$ 1.32), expressed dissatisfaction with health $(O R=1.22,1.13-1.33)$, and reduced performance of IADL $(O R=1.10,1.06-1.15)$ were associated with slow GS. The AUC value on $R O C$ analysis was 0.72 for the selected variables (Figure 2 in Supplementary Files).

\section{Discussion}

Walking is fundamental to maintaining health and independence in the older people. This study analyzes the association of slow GS with measures of health status revealed in a nationwide survey of a population of older ambulatory Chinese. The data in this study show that slow GS is associated with higher co-morbidity, increased prevalence of chronic diseases, greater depression, and lower physical and cognitive functions [11-13, 16-20]. The study confirms that a reduced GS implies a overall reduction in health status in older Chinese people [11-13, 16-20]. As a result, the medical cost in the slow GS group was much higher than that in the normal GS group. Specifically, the mean medical costs for men and women in the slow GS group were 9778 yuan (RMB) and 5893 yuan (RMB), respectively, compared to 4210 yuan (RMB) and 3196 yuan (RMB), respectively, in the normal GS group $(P<0.0001)$. The higher costs in the slow GS group may be the result of more medications $(P<0.0001)$, increased hospitalization $(P<0.0001)$, or increased need for care by others $(P<0.0001)$. The cost figures in this study confirm some data from earlier studies that also showed a relationship between reduced walking ability and higher medical costs [20]. In short, GS can be a quick, inexpensive, and useful indicator of older people who have chronic and expensive declines in health that might be circumvented by targeted intervention $[12,13]$.

The walking distance and the cutoff point for defining slow GS has varied in previous studies $[12,13]$. In our study, the difference between the mean GS of the slow and the normal GS groups, for each gender, was about $0.5 \mathrm{~m} / \mathrm{s}(1.0 \mathrm{~m} / \mathrm{s}$ vs. $0.5 \mathrm{~m} / \mathrm{s})$. The observed difference in GS may due to the difference in health status of the study subjects. Our study reveals a $10-20 \%$ increasing in the prevalence of common chronic diseases. A meaningful change in GS has been established at $0.1 \mathrm{~m} / \mathrm{s}$. Study finds that a decline in gait speed of $0.1 \mathrm{~m} / \mathrm{s}$ within 1 year increased the subsequent 5-year mortality rate [21]. In contrast, a GS increase of $0.1 \mathrm{~m} / \mathrm{s}$ has been associated with observable health benefits; e.g., an increase as a result of intervention has been associated with a reduction of $17.7 \%$ in the absolute risk of death [22], fewer hospitalization days (2.3 fewer days), and 1-year cost reductions of \$1,188 [20]. Additional outcomesbased studies hold the promise of providing more informed interpretations of GS values for use in evaluating the risk of adverse health outcome in the older people. 
The most important challenges of ageing are related to reduced physical and cognitive function. The associations between slow GS and measures of impaired cognitive function have been studied in many populations [16-18]. Our study confirms that slow GS is associated with reduced cognitive function. Up until now, a causal relationship has not been established but is considered to be bidirectional [23]. Furthermore, it is often observed that low physical performance, impaired cognitive function, and depression co-exist in older populations [24,25]. The association of slow GS with lower GDS scores has also been found in this study (Table 1 in Supplementary Files). Other factors such as "disinterest in life", "dissatisfaction with health status", which have negative psychological features, add more information about the association of slow GS and psychological status. A six-year cohort study has indicated that a state of depression affects physical health and impedes recovery from physical dysfunction [26]. Studies have shown that symptoms of depression are associated with not only lower physical functional performance but also cognitive decline, both of which are accelerated through negative feedback to further depression $[27,28]$.

The mutual associations of the cluster of low physical performance, cognitive function and depressed mood suggest that there is a path to gaining multiple benefits $[29,30]$. For example, there is evidence that increased engagement in daily activities has the potential to improve health and well-being of the older people [2]. Doing housework is a physically-demanding and intelligence-engaging activity that can generate a positive attitude towards life and a good psychological status. Interestingly, in this study we found that there were more people in the normal GS group doing housework (about 20\% more). Logistic regression revealed that doing housework showed a strong association with faster GS (Table 2 in Supplementary Files). Doing housework may help people maintain a normal GS through their active engagement in daily life, which, in turn, helps them maintain better cognitive and psychological functions. Similar to Woo's study in a population of older Chinese in Hong Kong, we found that alcohol intake was associated with faster walking speeds. This association may be explained by drinking people being more likely to have social activities and, as a result, being more likely to have better psychological status, which helps people maintain faster walking speeds and good health status [3].

This study has some limitations. Firstly, the Asia Working Group for Sarcopenia (AWGS) defines slow GS as $<0.8 \mathrm{~m} / \mathrm{s}$ (when walking $6 \mathrm{~m}$ in a straight line) [15]. The survey used in this study measured the walking speed over a distance of 20 meters, and inevitably, the walking speed will be lower due to the mid-course U-turn taken to return to the starting line. This situation may bring the limitation due to the fact that results of the different studies cannot be easily compared to each other. Secondly, as a crosssectional study, it is difficult to establish a cause-effect relationship between slow GS and poor health status. Thirdly, this study excluded from the analysis people who could not complete the walking measurement, which may introduce in a certain degree of bias. However, the main purpose of this study was to explore the relationship between walking speed and health-related conditions, and to decide whether GS could be used as an indicator of the health status in the older people.

\section{Conclusion}


This study confirms that in ambulatory older Chinese people, slow GS is associated with overall reduction in health status. People with slow GS should be targeted for possible early intervention. Encouraging older people to have a positive attitude towards life and to become more involved in daily life will help them maintain a better health status.

\section{List Of Abbreviations}

Gait speed (GS); the Geriatric Depression Scale (GDS); chronic obstructive pulmonary disease (COPD); activities of daily living (ADL); instrumental activities of daily life (IADL); Mini-mental State Examination (MMSE); the Asia Working Group for Sarcopenia (AWGS); receiver-operating characteristic (ROC).

\section{Declarations}

\section{Ethics approval and consent to participate}

All participants were informed about the study and assured of confidentiality in reporting the results. The consents were verbal and obtained before the investigation started. The study protocol and method of obtaining consent has been approved by Ethics Committee of Beijing Hospital, Ministry of Health.

\section{Consent for publication}

Not applicable.

\section{Availability of data and materials}

The datasets used and analyzed during the current study are available from the corresponding author on reasonable request.

\section{Competing interests}

The authors have no conflicts of interest to declare.

\section{Funding}

This study was funded by National Department Public Benefit Research Foundation, Ministry of Health P. R. China (No. 201302008). The funding body had no role in the design of the study; collection, analysis, and interpretation of data; and in writing the manuscript.

\section{Authors' Contributions}

PZ collected and analyzed the data, and wrote the paper. YLD assisted with analyzing the data and writing the paper. YWH, JP and HG collected the data. JL applied the fund, assisted with formulating the research question and designed the studies. TMZ has made contributions to the following: (1) the 
conception and design of the study, and interpretation of data, (2) revising the article critically for important intellectual content, (3) final approval of the version to be submitted.

\section{Acknowledgements}

The authors would like to thank the older people answering the questionnaires. The authors would also like to express thanks to all the 13 hospitals (Beijing Hospital, Beijing Xuanwu Hospital of Capital Medical University, Beijing Geriatric Medical Research Center, the People's Liberation Army General Hospital, Peking University Third Hospital, West China Hospital of Sichuan University, First Affiliated Hospital of Chongqing Medical University, First affiliated hospital of Xi 'an Jiaotong University, Xijin Hospital Fourth Military Medical University, Xiangya Hospital of Central South University, Third Xiangya College of Central South University, Ruijin Hospital Affiliated to Shanghai Jiao Tong University School of Medicine, First Affiliated Hospital of Harbin Medical University), who made contribution to data collections. Yi Zhang, Enyi Zhang, Yan Zhang, Yuan Miao and Jing Yang involved in the survey and data entry portions of this study. We would like to express our appreciation to Dr. Donald Barnes, South China University of Technology, for helpful comments on the final revision of this paper.

\section{References}

1. Rowe JW, Kahn RL. Successful aging. Aging, 1998, 10(2):142.

2.Ng SH, Cheung CK, Chong AM, Woo J, Kwan AY, Lai S. Aging well socially through engagement with life: Adapting Rowe and Kahn's model of successful aging to Chinese cultural context. Int J Aging Hum Dev 2011; 73: 313-330.

3.Woo J, Leung J, Zhang T. Successful Aging and Frailty: Opposite Sides of the Same Coin? J Am Med Dir Assoc. 2016. 17(9): 797-801.

4. Tanimoto Y, Watanabe M, Sun W, Tanimoto K, Shishikura K, Sugiura Y, Kusabiraki T, Kono K. Association of sarcopenia with functional decline in community-dwelling elderly subjects in Japan. Geriatr Gerontol Int 2013; 13, 4: 958-63.

5. Arango-Lopera VE, Arroyo P, Gutiérrez-Robledo LM, Pérez-Zepeda MU, Cesari M. Mortality as an adverse outcome of sarcopenia.J Nutr Health Aging 2013; 17: 259-262.

6. Cesari M, Pahor M, Lauretani F, Zamboni V, Bandinelli S, Bernabei R, Guralnik JM, Ferrucci L. Skeletal muscle and mortality results from the InCHIANTI Study. J Gerontol A Biol Sci Med Sci 2009; 64: 377-384.

7. Goodpaster BH, Park SW, Harris TB, Kritchevsky SB, Nevitt M, Schwartz AV, Simonsick EM, Tylavsky FA, Visser $\mathrm{M}$, Newman AB. The loss of skeletal muscle strength, mass, and quality in older adults: the health, aging and body composition study. J Gerontol A Biol Sci Med Sci 2006; 61:1059-1064. 
8. McCartney N, Hicks AL, Martin J, Webber CE. Long-term resistance training in the elderly: effects on dynamic strength, exercise capacity, muscle, and bone. J Gerontol A Biol Sci Med Sci. 1995;50: B97-104.

9. Liu CJ, Latham NK. Progressive resistance strength training for improving physical function in older adults. Cochrane Database Syst Rev 2009; CD002759.

10. Woo J. Walking speed: A summary indicator of frailty? J Am Med Dir Assoc 2015; 16: 635-637.

11. Cesari M. Role of gait speed in the assessment of older patients[J]. JAMA, 2011, 305(1): 93-94.

12. Peel NM, Kuys SS, Klein K. Gait Speed as a Measure in Geriatric Assessment in Clinical Settings: A Systematic Review. J Gerontol A Biol Sci Med Sci. 2012 January;68(1):39-46.

13. Abellan van Kan G, Rolland Y, Andrieu S, Bauer J, Beauchet O, Bonnefoy M, Cesari M, Donini LM, Gillette Guyonnet S, Inzitari M, et al. Gait speed at usual pace as a predictor of adverse outcomes in community-dwelling older people an International Academy on Nutrition and Aging (IANA) Task Force[J]. J Nutr Health Aging, 2009, 13(10): 881-889.

14. Cruz-Jentoft AJ. Bahat G. Bauer J. Boirie Y. Bruyère O. Cederholm T et al. Sarcopenia: revised European consensus on definition and diagnosis. Age Ageing. 2019; 48: 16-31.

15. Chen LK, Liu LK, Woo J, Assantachai P, Auyeung TW, Bahyah KS, Chou MY, Chen LY, Hsu PS, Krairit O, et al. Sarcopenia in Asia: consensus report of the Asian Working Group for Sarcopenia. J Am Med Dir Assoc. 2014. 15(2): 95-101.

16. Taniguchi Y, Yoshida H, Fujiwara Y, Motohashi Y, Shinkai S. A prospective study of gait performance and subsequent cognitive decline in a general population of older Japanese[J]. J Gerontol A Biol Sci Med Sci, 2012, 67(7): 796-803.

17. Verghese J, Wang C, Lipton RB, Holtzer R, Xue X. Quantitative gait dysfunction and risk of cognitive decline and dementia[J]. J Neurol Neurosurg Psychiatry, 2007, 78(9): 929-935.

18. Alfaro-Acha A, Al Snih S, Raji MA, Markides KS, Ottenbacher KJ. Does 8-Foot Walk Time Predict Cognitive Decline in Older Mexicans Americans?[J]. J Am Geriatr Soc, 2007, 55(2): 245-251.

19. Cawthon PM, Fox KM, Gandra SR, Delmonico MJ, Chiou CF, Anthony MS, Sewall A, Goodpaster B, Satterfield S, Cummings SR et al. Do muscle mass, muscle density, strength, and physical function similarly influence risk of hospitalization in older adults? J Am Geriatr Soc. 2009; 57(8): 1411-9.

20. Purser JL, Weinberger M, Cohen HJ, Pieper CF, Morey MC, Li T, Williams GR, Lapuerta P et al. Walking speed predicts health status and hospital costs for frail elderly male veterans. J Rehabil Res Dev. 2005, 42(4):535-546. 
21. Perera S, Studenski S, Chandler JM, Guralnik JM. Magnitude and patterns of decline in health and function in 1 year affect subsequent 5-year survival. J Gerontol A Biol Sci Med Sci. 2005; 60:894-900.

22. Hardy SE, Perera S, Roumani YF, Chandler JM, Studenski SA. Improvement in usual gait speed predicts better survival in older adults. J Am Geriatr Soc. 2007; 55:1727-1734.

23. Best JR, Liu-Ambrose T, Boudreau RM, Ayonayon HN, Satterfield S, Simonsick EM, Studenski S, Yaffe $\mathrm{K}$, Newman AB, Rosano $\mathrm{C}$, et al. An evaluation of the longitudinal, bidirectional associations between gait speed and cognition in older women and men. J Gerontol A Biol Sci Med Sci, 2016, 71(12): 1616-1623.

24. Hajjar I, Yang F, Sorond F, Jones RN, Milberg W, Cupples LA, Lipsitz LA. A novel aging phenotype of slow gait, impaired executive function, and depressive symptoms: relationship to blood pressure and other cardiovascular risks. J Gerontol A Biol Sci Med Sci. 2009. 64A(9): 994-1001.

25. Hsu YH, Liang CK, Chou MY, Liao MC, Lin YT, Chen LK, Lo YK. Association of cognitive impairment, depressive symptoms and sarcopenia among healthy older men in the veterans retirement community in southern Taiwan: a cross-sectional study. Geriatr Gerontol Int. 2014. 14 Suppl 1: 102-8.

26. Cronin-Stubbs D, de Leon CF, Beckett LA, Field TS, Glynn RJ, Evans DA. Six-year effect of depressive symptoms on the course of physical disability in community-living older adults. Arch Intern Med. 2000; 160 (20): $3074-3080$

27. Raji MA, Ostir GV, Markides KS, Goodwin JS. The interaction of cognitive and emotional status on subsequent physical functioning in older Mexican Americans: findings from the Hispanic established population for the epidemiologic study of the elderly. J Gerontol A Biol Sci Med Sci. $2002 ; 57$ (10): M678 - M682.

28. Apostolova LG, Cummings JL. Neuropsychiatric manifestations in mild cognitive impairment: a systematic review of the literature. Dement Geriatr Cogn Disord. 2008 ; 25 (2): 115 -126.

29. Kraft E. Cognitive function, physical activity, and aging: Possible biological linksand implications for multimodal interventions. Aging Neuro psychol Cogn 2012;19:248-263.

30. Atkinson HH, Rosano C, Simonsick EM, Williamson JD, Davis C, Ambrosius WT, Rapp SR, Cesari M, Newman AB, Harris TB, et al. Cognitive Function, Gait Speed Decline, and Comorbidities: The Health, Aging and Body Composition Study. J Gerontol A Biol Sci Med Sci, 2007, 62(8):844-850.

\section{Figures And Tables}

Due to technical limitations, figures 1 and 2 and table 1 and 2 are only available as a download in the supplemental files section.

\section{Supplementary Files}


This is a list of supplementary files associated with this preprint. Click to download.

- Table1.jpg

- Table2.jpg

- Figure1.jpg

- Figure2.jpg 